\title{
Optimizing accuracy of freehand cannulation of the ipsilateral ventricle for intracranial pressure monitoring in patients with brain trauma
}

\author{
Zhongyi Sun ${ }^{1}$, Lin $\mathrm{Wu}^{2}$, Zhixiong Liu ${ }^{1}$, Weiming Zhong $^{1}$, Zhifeng Kou ${ }^{3}$, Jinfang Liu ${ }^{1}$ \\ ${ }^{1}$ Department of Neurosurgery, Xiangya Hospital, Central South University, Changsha, China; ${ }^{2}$ Department of Ophthalmology and Anatomy and \\ Cell Biology, ${ }^{3}$ Departments of Biomedical Engineering and Radiology, Wayne State University, Detroit, MI, USA
}

Correspondence to: Zhifeng Kou, PhD. Departments of Biomedical Engineering and Radiology, Wayne State University, 818 W Hancock Street, Detroit, MI, 48201, USA. Email: zhifeng_kou@wayne.edu; Jinfang Liu, MD, PhD. Department of Neurosurgery, Xiangya Hospital, Central South University, 87 Xiangya Street, Changsha, 410008, China. Email: jinfang_liu@csu.edu.cn.

Background: Intracranial pressure (ICP) monitoring in traumatic brain injury (TBI) usually requires the placement of a catheter into the ipsilateral ventricle. This surgical procedure is commonly performed via a freehand method using surface anatomical landmarks as guides. The current accuracy of the catheter placement remains relatively low and even lower among TBI patients. This study was undertaken to optimize the freehand ventricular cannulation to increase the accuracy for TBI. The authors hypothesized that an optimal surgical plan of cannulation should give an operator the greatest degrees of freedom, which could be measured as the range of operation angle, range of catheter placement depth, and size of the target area.

Methods: An imaging simulation was first performed using the computed tomography (CT) images of 47 adult patients with normal brain anatomy. On the reconstructed 3D head model, four different coronal planes of ventricular cannulation were identified: a 4-cm anterior, a 2-cm anterior, a standard (central), and a 2 -cm posterior plane. The degrees of freedom during the cannulation procedure were determined, including the relevant angles, lengths of cannulation, cross-sectional area, and bounding rectangle of the lateral ventricle. Next, a retrospective assessment was performed on the CT scans of another 111 patients with TBI who underwent freehand ventricular cannulation for ICP monitoring. Postoperative measurements were also performed based on CT images to calculate the accuracy and safety of catheter placement between coronal planes in practice.

Results: Our simulation results showed that the $2-\mathrm{cm}$ anterior plane had more extensive degrees of freedom for ventricular cannulation, in terms of length of catheter trajectory ( $\%$ longer, $\mathrm{P}<0.001)$, crosssectional area of the lateral ventricle (14\% larger, $\mathrm{P}=0.046)$, and length of the lateral ventricle $(17 \%$ wider, $\mathrm{P}<0.001)$ than that of the standard plane, while both the $4-\mathrm{cm}$ anterior and $2-\mathrm{cm}$ posterior planes did not offer advantages over the standard plane in these ways. The mean length range of catheter trajectory in the 2 -cm anterior plane was 41 to $58 \mathrm{~mm}$. Retrospective assessment of TBI patients with ICP monitor placement also confirmed our simulation data. It showed that the accuracy of ipsilateral ventricle cannulation in the 2-cm anterior plane was $70.6 \%$, which was a significant increase from $42.9 \%$ in the standard plane $(\mathrm{P}=0.007)$.

Conclusions: Our imaging simulation and retrospective study demonstrate that different coronal planes could provide different degrees of freedom for cannulation, the 2-cm anterior plane has the greatest degrees of freedom in terms of larger target area and greater length range of the trajectory. The optimized surgical plan in this manner could improve cannulation accuracy and benefit a significant number of TBI patients.

Keywords: Traumatic brain injury (TBI); intracranial pressure (ICP); ventricular cannulation; surgical simulation

Submitted Jan 21, 2020. Accepted for publication Aug 06, 2020.

doi: 10.21037/qims-20-128

View this article at: http://dx.doi.org/10.21037/qims-20-128 


\section{Introduction}

Monitoring of intracranial pressure (ICP) in traumatic brain injury (TBI) patients usually requires placement of ICP transducer via fiberoptic or micro strain gauge devices placed in catheters. The most common placement sites of these transducers include the intraventricular space and brain parenchyma (1). Although parenchymal ICP provides equivalent, statistically similar pressure measurement compared to ventricular ICP in all cases and clinical settings $(1,2)$, conventional opinions have suggested that the ventricular ICP represents a more global and representative measurement of pressure throughout the intracranial space $(3,4)$. Additionally, ventricular drainage accompanied by ICP monitoring has also become generally accepted as a valuable adjuvant in the management of refractory ICP $(2,5)$. Severe TBI is currently one of the most common indications for ventricular cannulation for ICP monitoring and simultaneous cerebrospinal fluid (CSF) drainage. Still, it also represents the most challenging concern in terms of accurate catheter placement. Previous studies using image-based neural navigation systems (6-16) reported an improved placement accuracy $(17,18)$. However, they usually require the registration between the images and the patient's position. These methods are time-consuming and expensive. Further, the navigation systems have not been widely accessible in many developing or under-developed countries and regions $(4,19,20)$. As a result, the freehand method using surface anatomical landmarks remains the most common and standard method for ventricular cannulation (4,20-23).

Ventricular cannulation, also called ventriculostomy or external ventricular drain (EVD) placement, is also one of the most common neurosurgical procedures performed via a freehand method using surface anatomical landmarks as guides $(24,25)$. During this procedure, the catheter starts its trajectory from a surface anatomical landmark, Kocher's point (21), and follows a straight line determined by the intersection of two spatial planes: the sagittal plane through the ipsilateral medial canthus (IMC), and the coronal plane through two surface anatomical landmarks represented by the bilateral external auditory meatus (EAM). When the catheter tip reaches the lateral ventricle, CSF usually flows out of the catheter tail, and the ICP value is shown on a monitoring device, indicating that the procedure has been successfully accomplished (10). A detailed illustration of the procedure is shown in Figure 1.

Improved device technology has reduced failures and inaccuracies due to inherent problems. Still, the success rate of ventricular cannulation remains relatively low, ranging from $39 \%$ to $60 \%$ and even lower among TBI patients with a slit or atypical ventricle, or midline shift $(11,21,22,26-30)$. One possible reason is the origination of the surface anatomical landmarks utilized by the surgeons during operation (31), including Kocher's point, IMC, and EAM. Some of these points are derived from previous experience or knowledge rather than from a detailed analysis of the optimal angle or length of the trajectory. Previous studies assessing the relative optimal solution of different trajectories have largely focused on the varying sagittal directions of cannulation determined by several anatomical landmarks $(32,33)$, such as IMC, contralateral medial canthus (CMC), and nasion. However, knowledge regarding another equally important factor for targeting at the lateral ventricle, the coronal direction determined by EAM or other landmarks, was less reported.

In this paper, we first performed an imaging simulation and then conducted a retrospective study to understand the relationship between cannulation accuracy and different coronal directions of trajectory. We hypothesized that an optimal surgical plan of cannulation should give an operator the greatest degrees of freedom, which could be measured as the range of operation angle, range of catheter placement depth, and size of the target area.

\section{Methods}

This retrospective study was approved by the Medical Research Ethics Committee of Xiangya Hospital, Central South University (No.201307371). Written informed consents were obtained from legally authorized representatives for all participants that their data might be used for future retrospective studies. All subjects' personal identification information has been removed. A group sample of 47 patients was randomly selected from the radiograph dataset with normal brain reports, regardless of their purpose of seeking medical care. CT images of these subjects were used to perform an imaging simulation to build a surgical plan of cannulation. Then, 111 patients with severe TBI who had cannulation for ICP monitoring were retrospectively evaluated to validate our proposed surgical plan.

\section{Imaging simulation}

Head CT data were collected using thin-cut $(1 \mathrm{~mm}$ thickness, $512 \times 512$ matrix) CT scans (Siemens, Erlanger, 


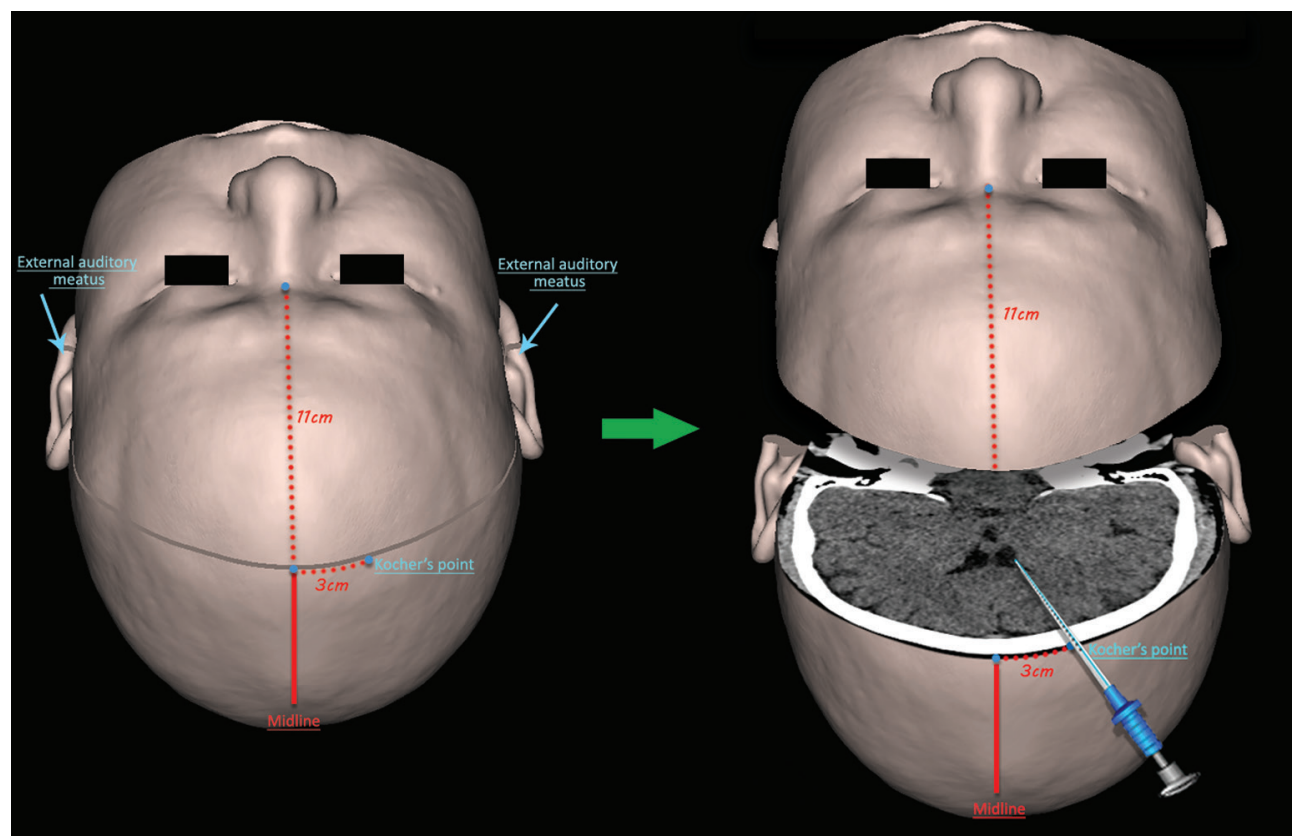

Figure 1 Volume-rendered CT scan of a patient with normal anatomy. On the left side of the figure, Kocher's point, and both external auditory meatus (EAM) were marked on the scalp. Kocher's point was approximately $11 \mathrm{~cm}$ above the nasion and $3 \mathrm{~cm}$ from the midline. On the right side of the figure, a coronal plane was reconstructed from three non-collinear points, Kocher's point, and both EAM. Starting from Kocher's point, the cannulation was simulated in this coronal plane with the target in the ipsilateral ventricle.

Germany) of 47 randomly selected adult outpatients with normal head structures. Mimics software (Materialise company, Leuven, Belgium) was used to reconstruct the 2D CT slices into 3D.

To build up a surgical plan of ventricular cannulation from CT data, a specific coronal plane was first determined by three non-collinear surface landmarks. The standard (central) coronal plane was produced using these three non-collinear points, Kocher's point and a pair of EAM. These points were previously marked on the surface of the scalp and then were used as reference points to reconstruct a coronal plane using multiplanar reconstruction (MPR) (Figure 1, left). Next, the desired trajectory was determined in this coronal plane, starting from Kocher's point and ending in the ipsilateral ventricle (Figure 1, right). Similarly, to acquire three more coronal planes that all shared the Kocher's point, three more pairs of points were marked on the scalp as well, located horizontally behind and in front of the EAM with the separation distance of $2 \mathrm{~cm}$ (Figure 2). Two centimeters was chosen because surgeons often use a thumb-width (almost $2 \mathrm{~cm}$ in a typical person) as an arbitrary measurement tool during operations. Then these four coronal planes, the 4-cm anterior (to EAM, the same below) plane, the 2-cm anterior plane, the standard (central) plane, and the $2-\mathrm{cm}$ posterior plane could be reconstructed through these points.

The parameters related to the cannulation in each coronal plane were measured using the same software. Our rationale was that the optimal coronal plane would provide the widest-angle range (from maximum to minimum), longest length range (the difference between the longest length and shortest length), and greatest area (see Figure 3). As such, the operator would have the greatest degrees of freedom and higher accuracy. Using Kocher's point as the vertex of an angle, the difference between the maximum angle $(\max \mathrm{A})$ and the minimum angle $(\min \mathrm{A})$ that provided successful cannulation was measured (34). The maximum angle was defined as the angle producing a catheter trajectory along the medial edge of the lateral ventricle to the midline, while the minimum angle was defined as the angle producing a catheter trajectory along the lateral edge of the lateral ventricle to the midline (Figure $3 A$ ). Furthermore, the length range of a successful trajectory could be calculated by subtracting the shortest entry length (SL) from the longest exit length (LL) (35), which were the distance from the dural entry point to the nearest and the farthest 


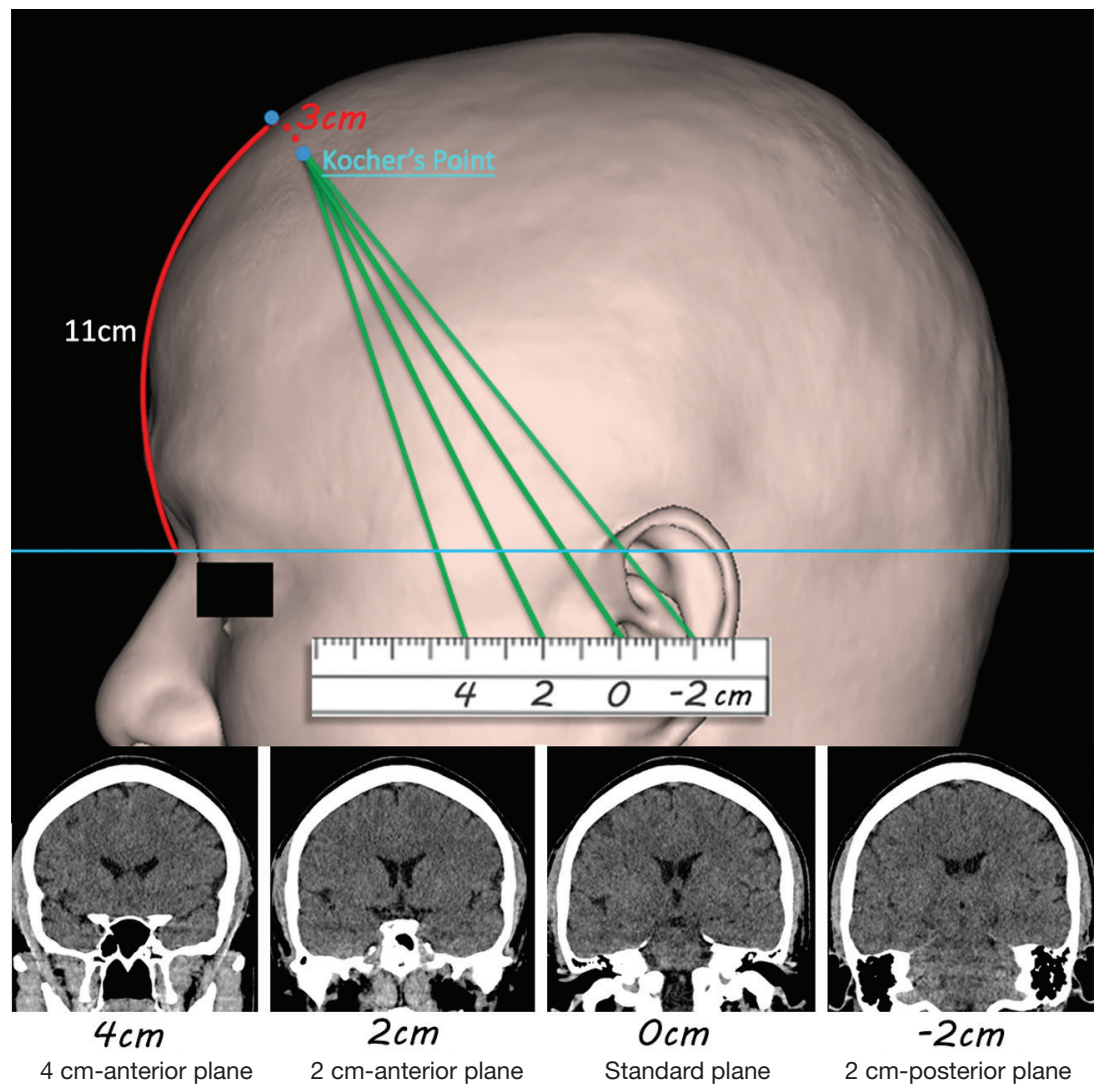

Figure 2 The upper figure shows the sagittal view of the volume-rendered head. Using the external auditory meatus (EAM) as the point of origin $(0 \mathrm{~cm})$, three other landmark points and their contralateral points could be acquired on either side of the head, anterior and posterior to the external auditory meatus with different separation distances $(4 \mathrm{~cm}, 2 \mathrm{~cm}$ and $-2 \mathrm{~cm})$. A defined horizontal line in the figure is passing through the nasion and external occipital protuberance. Below the volume-rendered head is shown the multiplanar reconstructions of the four different coronal planes represented by the green lines above.

edge of the ipsilateral ventricle, respectively (Figure 3B). In addition, in each coronal plane, the cross-sectional area of the lateral ventricle representing the possible target field was measured (Figure 3C). To describe the geometric morphology of the lateral ventricle in the plane, a bounding rectangle was drawn closely around it, and the vertical and horizontal length of this rectangle was recorded (Figure 3C).

\section{Clinical assessment}

The second part of this study was a retrospective clinical study to evaluate the accuracy and safety of trajectories in the standard plane and other coronal planes. Patients suffering from severe closed TBI who underwent freehand ventricular cannulation for ICP monitoring in the Neurosurgery Department of Xiangya Hospital, Central South University, China, between 2015 and 2016 were studied.

To reduce the influence of difference of the mean bicaudate index (BCI) between the imaging simulation study and clinical study, patients who experienced intracranial mass lesion evacuation and/or decompressive craniectomy were excluded. These excluded patients generally have a slit or atypical ventricle, or midline shift. The data from the 111 patients who solely underwent the surgical operation of ICP monitoring during the hospitalization were included (Table 1). Seventeen of 111 (15.3\%) patients had CT scan of midline shift less than $5 \mathrm{~mm}$. Placement of a ventricular catheter with a built-in transducer (Codman, New Brunswick, US or Sophysa, Orsay, France) was performed in freehand style by experienced staff in an operating room (OR) under general anesthesia (36). Each case had 1-3 attempts to be supposed to achieve CSF flow from the lateral ventricle; an additional attempt was avoided from 

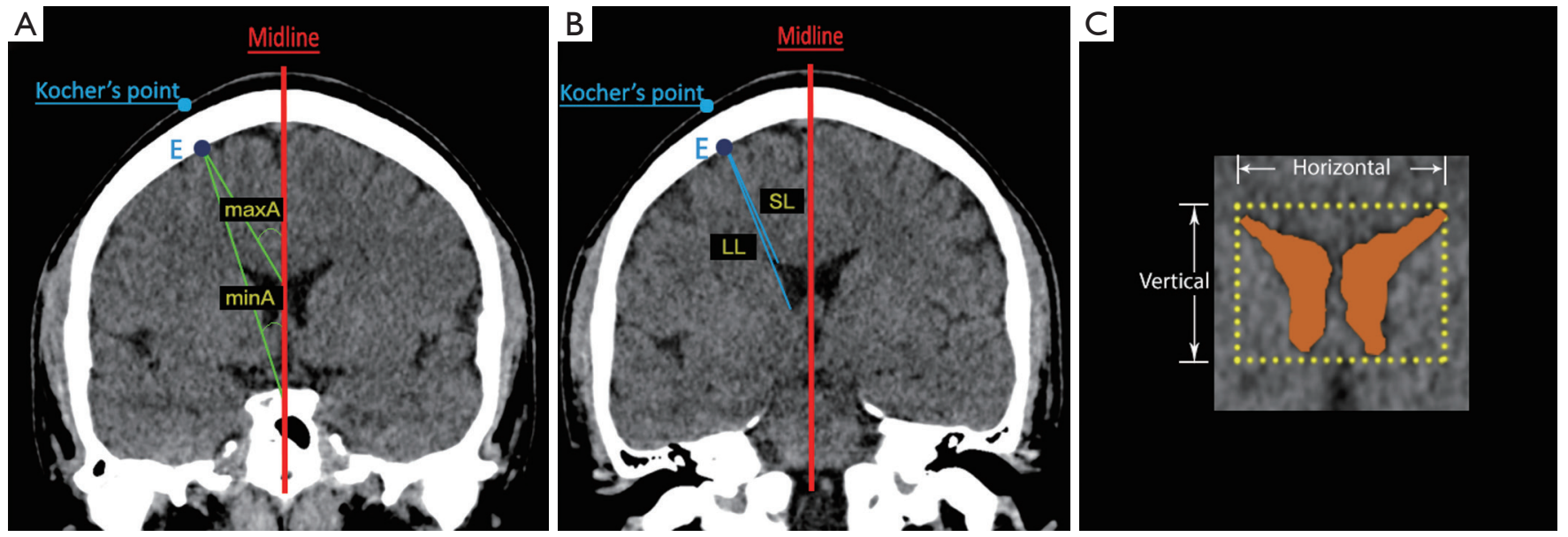

Figure 3 The degrees of freedom during the cannulation procedure. (A) The maximum and minimum angles and (B) the short and long lengths of ventricular cannulation demonstrated in one example plane. (C) The cross-sectional area and the bounding rectangle (dotted line) of the lateral ventricle. maxA, maximum angle; minA, minimum angle; LL, longest exit length; SL, shortest entry length; E, entry point of the dura corresponding to Kocher's point on the scalp.

Table 1 Clinical data on 111 patients with TBI

\begin{tabular}{lccc}
\hline & Overall & Standard plane & 2-cm anterior plane \\
\hline Patients (number) & 111 & 77 & 34 \\
Age (years) & 48.5 & 48.3 & 49.4 \\
Gender (M/F) & $85 / 26$ & $52 / 25$ & $25 / 9$ \\
Admission diagnosis, $\mathrm{n}$ [\%] & & & $37[48]$ \\
Contusion & $51[46]$ & $26[34]$ & $14[41]$ \\
SDH & $38[34]$ & $9[12]$ & $12[35]$ \\
IVH & $13[12]$ & $5[6]$ & $4[12]$ \\
SAH & $9[8]$ & $4[12]$ & \\
\hline
\end{tabular}

TBI, traumatic brain injury; SDH, subdural hematoma; IVH, intraventricular hemorrhage; SAH, subarachnoid hemorrhage.

causing unnecessary brain insult. The case without CSF flow after multiple attempts probably indicated that the tip of the catheter was located within brain parenchyma other than ventricular spaces (including the ipsilateral, contralateral, and 3rd ventricle). Because parenchymal ICP is statistically proved similar to ventricular ICP, these cases had not been revised until the use of CSF drainage was a necessary valuable means to control refractory ICP.

Among the 111 patients who had ventricular cannulation, there were 51 cases (46\%) with acute cerebral contusion, 38 cases (34\%) with acute subdural hematoma (SDH), 13 cases (12\%) with intraventricular hemorrhage (IVH), and 9 cases (8\%) with subarachnoid hemorrhage (SAH). Regarding the side of cannulation, 81 out of 111 (73\%) had cannulation on the right side and $30(27 \%)$ on the left side as determined by the clinical situation. Regarding the choice of coronal plane for cannulation, 77 out of 111 patients used the current standard procedure in the standard coronal plane, and 34 patients used the $2-\mathrm{cm}$ anterior coronal plane to cannulate the ipsilateral ventricle. Postoperative CT was performed in all patients to confirm accuracy and safety.

\section{Statistical analysis}

Results are expressed as the mean \pm standard deviation (SD). Frequencies and percentages are presented for categorical 
variables. Normal distribution and homogeneity of variances were tested using the Shapiro-Wilk and Levene method, respectively. In the imaging simulation portion of the study, the significance of differences between the four groups was evaluated by one-way analysis of variance (ANOVA) followed by Dunnett's test for multiple comparisons. In the retrospective clinical part, differences between groups were assessed by the Chi-Square test. Analyses were done with SPSS (IBM, NY, US) statistical analysis software. Tests were two-tailed tests, and $\mathrm{P}$ values less than 0.05 were considered significant after Bonferroni correction.

\section{Results}

In the imaging simulation with normal CT data from 47 patients, the mean BCI was $0.141 \pm 0.128$, which was the ratio of the width of both lateral ventricles to the distance between the inner tables of the skull at the level of the head of the caudate nucleus. After a total of 188 coronal planes were reconstructed by MPR, the simulation of ventricular cannulation was performed on the right side of the brain in each coronal plane, which is the default side for operating because it does not control language function. During this process, the cross-sectional area, minA, maxA, angle range, SL, LL, and length range were measured. The data from the standard plane served as the control group, and a Dunnett's test was used after the ANOVA to identify the pairs with significant differences (Figure 4). The crosssectional area of the bilateral ventricles, representing the target field of ventricular cannulation, was larger in the standard plane than in the $4-\mathrm{cm}$ anterior and $2-\mathrm{cm}$ posterior plane, but $14 \%$ smaller than in the $2-\mathrm{cm}$ anterior plane $\left(189.2 \pm 59.6\right.$ vs. $\left.215.4 \pm 53.5 \mathrm{~mm}^{2}, \mathrm{P}=0.046\right)$. The maxA, the $\min \mathrm{A}$, and the angle range did not achieve statistical significance between the $2-\mathrm{cm}$ anterior and the standard plane. The 4-cm anterior plane had the smallest angle range of ventricular cannulation among these four planes. The LL and the length range in the $2-\mathrm{cm}$ anterior plane were both longer than that in the standard plane, while the SL had no significant difference among these four planes. The vertical distance of the bounding rectangle closely around the lateral ventricle was $17 \%$ greater in the $2-\mathrm{cm}$ anterior plane than in the standard plane $(17.37 \pm 2.91$ vs. $14.88 \pm 2.64 \mathrm{~mm}$, $\mathrm{P}<0.001$ ), but the horizontal distance was not statistically significant. Because the $4-\mathrm{cm}$ anterior and $2-\mathrm{cm}$ posterior planes did not offer advantages over the standard plane, specific comparisons between only the 2 -cm anterior plane and the standard plane are demonstrated in Table 2.
In the retrospective study of 111 TBI patients' data, each patient had only one catheter placed during the hospitalization. The postoperative CT scan showed that 57 catheter tips were successfully placed into the ipsilateral ventricle, 7 in the contralateral ventricle, 10 in the third ventricle, and 37 in extra-ventricular spaces (Table 3). These 37 cases were not revised after the postoperative CT scan because either ventricular or parenchymal ICP could serve for the same purpose of ICP monitoring (37). Cannulation was performed in the standard plane in 77 of 111 patients; of these, only 33 cannulas were placed into the ipsilateral ventricle, yielding an accuracy of ipsilateral ventricle placement of $42.9 \%$ (33/77). Cannulation was performed in the 2-cm anterior plane for 34 patients, 24 of whom had cannula placement into the ipsilateral ventricle, yielding an accuracy of $70.6 \%$ (24/34) (Figure 5). Our evaluation criterion for accuracy was more rigid than other standards because it was defined by whether the catheter tip was located inside the ipsilateral ventricle. The mean BCI of these 111 patients was $0.129 \pm 0.082$ and had no significant difference with our previous imaging simulations $(0.129 \pm 0.082, \mathrm{~N}=111$, vs. $0.141 \pm 0.128, \mathrm{~N}=47, \mathrm{P}=0.517)$. The burr hole location, which is the distance between midline and the center of the burr hole on the outer surface of the skull, was $2.71 \pm 0.33 \mathrm{~cm}$, having no significant difference with $3 \mathrm{~cm}(\mathrm{P}=0.09)$. A total of 5 patients $(4.5 \%)$ demonstrated hemorrhage along the catheter path on postoperative CT scans, and none of them was significant hemorrhage; 3 of these $(3.9 \%)$ were cannulated using the standard plane and $2(5.9 \%)$ using the 2 -cm anterior plane.

\section{Discussion}

The question addressed by the present study was whether there was an optimal surgical plan for ventricular cannulation, which could give an operator the greatest degrees of freedom, leading to a better probability of successful cannulation. The main finding of our study was that the $2-\mathrm{cm}$ anterior plane is a more optimal plane for cannulation with a larger target area and greater length range than the standard coronal plane used most often today. The accuracy of cannulation in this new coronal plane has been increased by about $28 \%$, as demonstrated in patients cannulated in this study.

\section{Determination of optimum operating plane}

In the imaging simulation part, we found that cannulation 

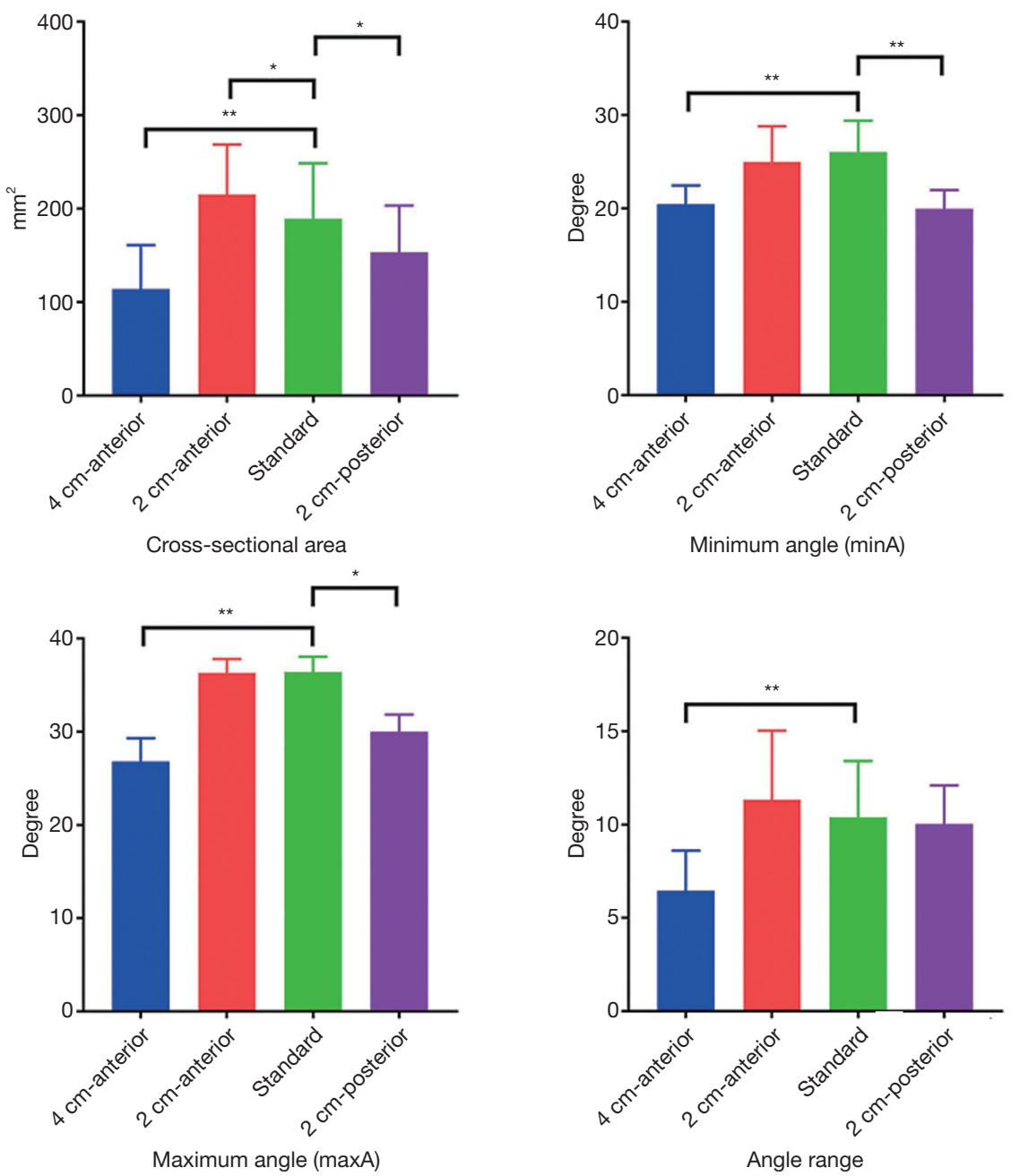

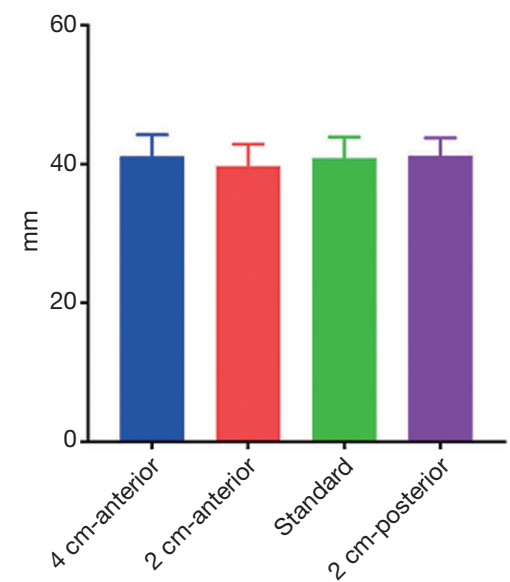

Shortest entry length (SL)

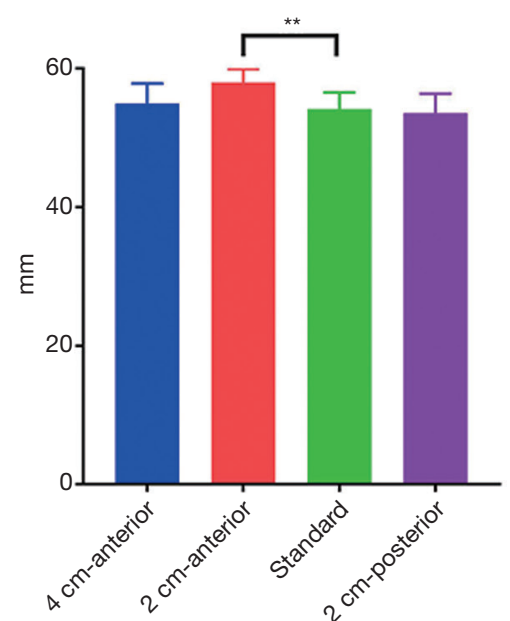

Longest exit length (LL)

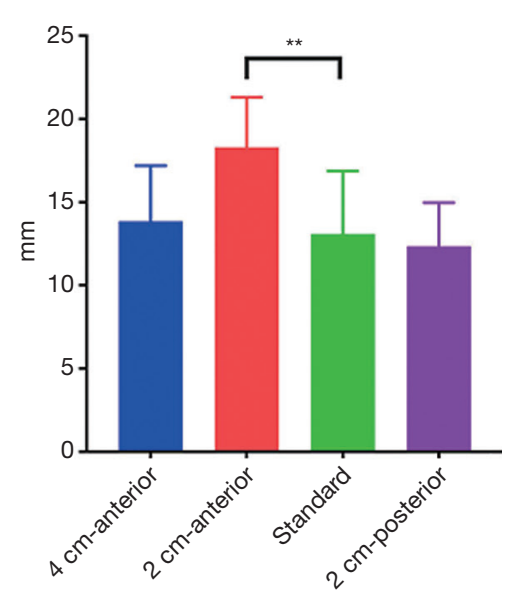

Length range

Figure 4 Parameters of cannulation simulation were measured in different coronal planes. The standard plane served as a baseline to compare with other three coronal planes by the Dunnett's test, respectively. The 2-cm anterior plane had larger cross-sectional area and greater length range than the standard plane. The angle range between the $2-\mathrm{cm}$ anterior and the standard plane had no statistical difference. *, $\mathrm{P}<0.05 ;{ }^{* *}, \mathrm{P}<0.01$. 
Table 2 Summary of imaging simulation between the $2 \mathrm{~cm}$-anterior and the standard plane

\begin{tabular}{lccc}
\hline & 2cm-anterior plane & Standard plane & P value \\
\hline Area of LV & $215.37 \pm 53.46$ & $189.22 \pm 59.64$ & $0.046^{*}$ \\
maxA & $36.32 \pm 1.50$ & $36.42 \pm 1.63$ & 0.988 \\
minA & $24.98 \pm 3.83$ & $26.03 \pm 3.38$ & 0.196 \\
Range of angle & $11.34 \pm 3.70$ & $10.39 \pm 3.02$ & 0.246 \\
LL & $58.01 \pm 1.93$ & $54.16 \pm 2.40$ & $<0.001^{*}$ \\
SL & $39.71 \pm 3.17$ & $41.07 \pm 3.74$ & 0.102 \\
Range of length & $18.29 \pm 3.02$ & $13.09 \pm 3.79$ & $<0.001^{*}$ \\
Bounding rectangle & & & $<0.001^{*}$ \\
Vertical & $17.37 \pm 2.91$ & $22.73 \pm 5.07$ & 0.217 \\
Horizontal & $24.06 \pm 5.28$ & $14.88 \pm 2.64$ &
\end{tabular}

${ }^{*}$, statistically significant $\mathrm{P}$ values. LV, lateral ventricle; maxA, maximum angle; minA, minimum angle; LL, longest exit length; SL, shortest entry length.

Table 3 Summary of clinical study between the $2 \mathrm{~cm}$-anterior and the standard plane

\begin{tabular}{lccc}
\hline Variables & Total & 2-cm anterior plane & Standard plane \\
\hline Cases & 111 & 34 & 77 \\
BCl & $0.129 \pm 0.082$ & $0.127 \pm 0.067$ & $0.130 \pm 0.088$ \\
Location of catheter tip: $\mathrm{n}(\%)$ & & & \\
Ipsilateral V. & $57(51.4)$ & $24(70.6)$ & $33(42.9)$ \\
Contralateral V. & $7(6.3)$ & $3(8.8)$ & $4(5.2)$ \\
Third V. & $10(9.0)$ & $2(5.9)$ & $8(10.4)$ \\
Extra-ventricular & $37(33.3)$ & $5(14.7)$ & $32(41.6)$ \\
Accuracy: \% & 51.4 & 70.6 & 42.9 \\
Postoperative Hemorrhage: $\mathrm{n}(\%)$ & $5(4.5)$ & $2(5.9)$ & $3(3.9)$ \\
\hline
\end{tabular}

${ }^{*}$, statistically significant $\mathrm{P}$ values. $\mathrm{BCl}$, bicaudate index; $\mathrm{V}$., ventricle.

in the 2-cm anterior plane offers improvements in several parameters that could make the catheter tip more likely to be accurately placed into the ipsilateral ventricle. By measuring these geometric parameters among the four coronal planes investigated here, we detected two primary parameters which could explain the advantages of the $2-\mathrm{cm}$ anterior plane. One advantage is the increased length range for successful cannulation. The LL of ventricular cannulation was $7 \%$ greater in the $2-\mathrm{cm}$ anterior plane than the standard plane, while the SL was similar, demonstrating a greater length range that will result in successful placement within the ventricle. Another observed advantage is the cross-sectional area of the lateral ventricle, which is about $14 \%$ larger in the $2-\mathrm{cm}$ anterior plane than in the standard plane. The increased cross-sectional area in the target field of ventricular cannulation means that there is a greater probability of placing a catheter tip into the lateral ventricle.

\section{Degrees of freedom}

Ventricular cannulation is a core neurosurgical procedure that is commonly performed by young doctors. As these young doctors gain experience, the accuracy of their 


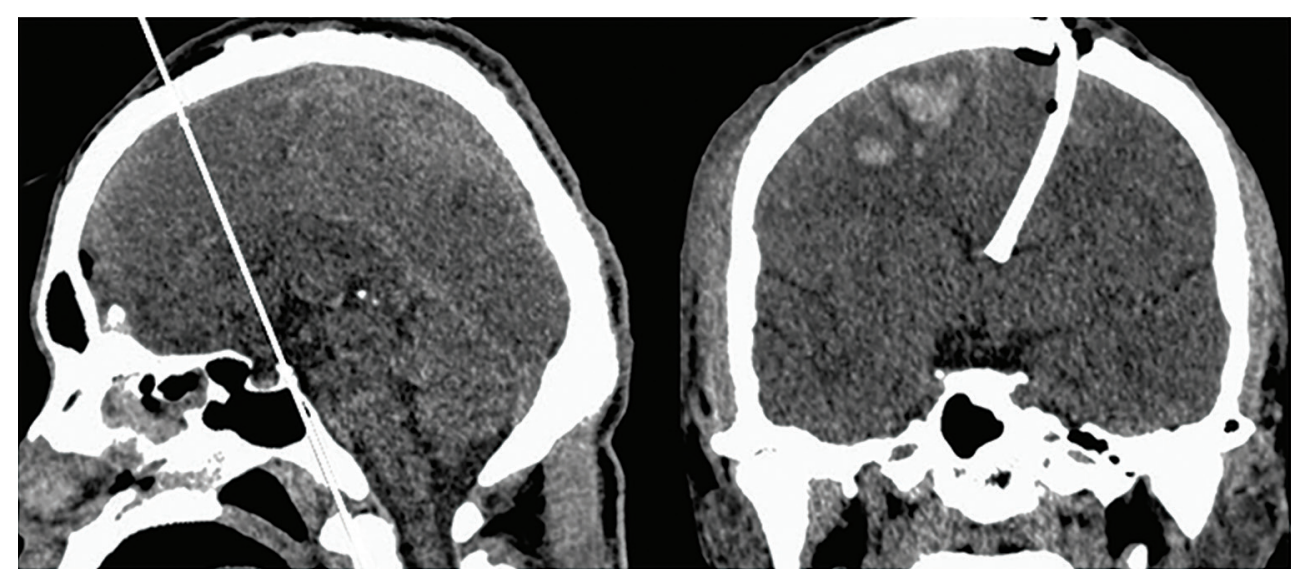

Figure 5 The postoperative CT scan of a traumatic brain injury (TBI) patient. The left part of the figure shows the 2-cm anterior plane (white line) on the sagittal view of the CT scan. On the right, an intracranial catheter for intracranial pressure (ICP) monitoring and cerebrospinal fluid (CSF) drainage placed directly into the ipsilateral ventricle in the 2-cm anterior plane.

cannulation would be expected to improve $(4,28,30)$. Practicing with more degrees of freedom for cannulation may indicate a favorable case for catheter placement accuracy in which the target field is physically larger. Once the coronal plane has been confirmed, the angle and length of cannulation determine the probability of successful placement of the catheter into the ipsilateral ventricle. The angle of cannulation depends on the width of the lateral ventricle in this coronal plane and ranges from the minimum angle to the maximum angle. The length of cannulation relies on the height of the lateral ventricle. The length range is ideally shorter than the longest exit length and longer than the SL (Figure 3). In our study, there was no difference in either the maximum or minimum angle of cannulation between the $2-\mathrm{cm}$ anterior and the standard plane. However, the longest exit length between these two planes did show a difference. It implied that the lateral ventricle probably had a morphological difference between the two planes. A bounding rectangle closely drawn around the lateral ventricle demonstrated that the vertical distance of the lateral ventricle in the $2-\mathrm{cm}$ anterior plane was $17 \%$ greater than that in the standard plane, while the horizontal distance had no difference. This morphological difference suggests that the catheter tip has a greater probability of being successfully placed in the vertical direction in the $2-\mathrm{cm}$ anterior plane.

Our study showed that the recommended length range of cannulation in the $2-\mathrm{cm}$ anterior plane was 41 to $58 \mathrm{~mm}$, which was the mean value calculated by normal CT data. The length range of cannulation could be derived from the actual measurement in the $2-\mathrm{cm}$ anterior plane after the multiplanar reconstruction of CT scans for each patient. It would be more accurate than the mean value because of individual differences between patients. During ventricular cannulation, we suggest regarding the length range as the safe range to cannulate. If the path of cannulation was greater than $58 \mathrm{~mm}$ or the actual patient's value, the catheter tip might depart from the ipsilateral ventricle and end up in the contralateral, or third ventricle, or extraventricular space. This length range seemed less than earlier studies $(21,35)$, but had greater consistency than these previous studies because of the difference in the definition of length range of cannulation. Previous studies used the skull surface as the starting point of the length, while our study used the dural surface because this was a convenient way for operators to identify the scale of a catheter during surgical operations. The difference in our reported length of cannulation and that of previous studies is approximately the thickness of the skull, as would be expected from this difference in measuring.

\section{Choice of definition for accuracy}

The accuracy of ventricular cannulation in the $2-\mathrm{cm}$ anterior plane was shown to be increased compared to the standard plane in our retrospective clinical study. The definition of the accuracy of ventricular cannulation varies in different studies and their procedural purposes. Depending on varied purposes, ventricular cannulation could have different requirements than that of the measurement 
of ICP. Kakarla et al. (28) and Hayhurst et al. (38) proposed a 3 -grade score system based on the position of the catheter tip. O'Leary et al. (39) and Huyette et al. (21) defined the accuracy by measurement of the distance between the interventricular foramen and the catheter tip. Bogdahn et al. (40) thought the accuracy should be defined by the effectiveness of CSF drainage. In contrast, our study focused on improving the current freehand method to place the catheter tip directly into the ipsilateral ventricle rather than other brain structures, such as the contralateral ventricle, third ventricle, interhemispheric fissure, or other extra-ventricular spaces. Therefore, we defined the accuracy by whether the catheter tip was located inside the ipsilateral ventricle. Obviously, this accuracy seems more stringent than other evaluation criteria of earlier studies. If we revaluated their data following our criterion, the accuracy of ipsilateral ventricle cannulation would vary from $39 \%$ to $60 \%(11,21,22,26-30)$. However, all of these data included hydrocephalus patients with a BCI of more than 0.19 , which means the patients have expanded ventricle systems $(39,41)$. In these patients, enlarged ventricles would mean an increased target and facilitate successful cannulation, leading to an overestimation of the accuracy when applied to patients with normal BCI. Our results in trauma patients with a normal BCI of about 0.13 showed that the accuracy of ipsilateral ventricle cannulation was $42.9 \%$ when using the standard plane and $70.6 \%$ when using the $2-\mathrm{cm}$ anterior plane, representing a noticeable improvement.

\section{Iatrogenic bemorrbage during cannulation}

Intracranial hemorrhage along the catheter path during cannulation is a well-recognized complication, which should deserve attention as equally as cannulation accuracy. However, most imaging simulation studies have a primary outcome of ventricular catheter tip location, while the corresponding complication may be considered as a second outcome. Reported hemorrhage rates are widely variable from different studies, ranging from $1 \%$ to $41 \%(42,43)$, of which two-thirds were associated with misplaced catheters (44). These data underscore a need to optimize cannulation techniques to safe catheter placement. Our data demonstrated the cannulation in the 2-cm anterior plane was as safe as in the standard plane, without any increase in intracranial hemorrhage risk. During cannulation in our study, operators generally identify major cortical vessels beneath the dura through naked eyes, which remains the principal method used to avoid vascular injury. Robertson et al. (45) recently reported an imaging simulation study using CT angiography (CTA) (46) with venography (CTV) data to identify potential cortical vessels, which suggested a method might lessen the risk of intracranial hemorrhage during cannulation in theory. It may be possible to implement our optimized surgical plan accompanied by CTA/CTV data in the ventricular cannulation, in this manner, it could improve accuracy and simultaneously reduce iatrogenic hemorrhage.

\section{Clinical applications}

To practice the optimized surgical plan as this study presented, only a slight change is needed: making a thumbwidth $(2 \mathrm{~cm})$ forward movement from the EAM to mark a 2-cm anterior EAM point as the reference point of the coronal plane. Then starting with Kocher's point, imagine an intersection line of the sagittal and coronal plane as a trajectory for cannulation. This method could provide not only higher accuracy of cannulation than the conventional method into the ventricle, but also potential drainage of CSF from the ventricular structure, if necessary, to help relieve the ICP in severe brain trauma patients. This new method could also be widely used among patients with normal BCI, not only trauma patients but also other patients who need ventricular ICP monitoring or CSF drainage.

\section{Limitations and future work}

Kocher's point we chose in this study is widely used in literature $(11 \mathrm{~cm}$ posterior to nasion and $3 \mathrm{~cm}$ lateral to the midline), although different definitions of Kocher's point exist $(23,25,32)$. The use of a fixed entry point (burr hole) may be perceived as a limitation. The surgical plan mainly depends on the entry point, but in practice during the cannulation, this anatomical landmark is not necessarily to be used by all operators. We still need to consider other entry points to enhance robustness, such as mid-pupillary line and $2 \mathrm{~cm}$ from the midline. Another limitation is that we focused only on normal BCI patients. It lacks another group of severe TBI patients with a slit or atypical ventricle, or midline shift. As a result, the result of this study will only apply to TBI patients with normal BCI. Furthermore, the conditions under which the freehand cannulation performed was in the OR. Though OR could better avoid environmental contamination, we still need to consider other complex conditions in the future, like at the bedside 
in the emergency department or intensive care unit.

This is a retrospective study that could provide limited evidence to support our surgical plan proposed by the imaging simulation. In the future, the results can be significantly enhanced by a well-designed prospective study by including different cohorts of patients for various procedures, in a larger scale: ICP monitoring for severe TBI patients, EVD for IVH, and ventriculoperitoneal shunt for hydrocephalus. Further to test the accuracy of our proposed freehand operation, it will also be ideal to compare it with surgical navigation-assisted operations in prospective studies.

\section{Conclusions}

This study centered on the effect of different anatomical landmarks to build up an optimized surgical plan for cannulation. Our imaging simulation and retrospective study demonstrate that different coronal planes could provide different degrees of freedom for cannulation, the $2-\mathrm{cm}$ anterior plane has the greatest degrees of freedom in terms of larger target area and greater length range of the trajectory. The optimized surgical plan in this manner could improve cannulation accuracy and benefit a significant number of TBI patients.

\section{Acknowledgments}

We would like to thank Dr. Youming Zhang of the Radiology Department, Central South University, for his assistance with the Picture Archiving and Communication System (PACS), Ms. Furong Zhang for facilitating patient identification and collection of demographic information from the retrospective study period, and Dr. Natalie Wiseman and PhD candidate Malak Damdoum from Wayne State University for proofreading the manuscript to improve its readability.

Funding: The research was supported by a grant from the Xiangya medical big data Foundation.

\section{Footnote}

Conflicts of Interest: All authors have completed the ICMJE uniform disclosure form (available at http://dx.doi. org/10.21037/qims-20-128). The authors have no conflicts of interest to declare.

Ethical Statement: This retrospective study was approved by the Medical Research Ethics Committee of Xiangya Hospital, Central South University (No.201307371). Written informed consents were obtained from legally authorized representatives for all participants that their data might be used for future retrospective studies. All subjects' personal identification information has been removed.

Open Access Statement: This is an Open Access article distributed in accordance with the Creative Commons Attribution-NonCommercial-NoDerivs 4.0 International License (CC BY-NC-ND 4.0), which permits the noncommercial replication and distribution of the article with the strict proviso that no changes or edits are made and the original work is properly cited (including links to both the formal publication through the relevant DOI and the license). See: https://creativecommons.org/licenses/by-nc-nd/4.0/.

\section{References}

1. Vender J, Waller J, Dhandapani K, McDonnell D. An evaluation and comparison of intraventricular, intraparenchymal, and fluid-coupled techniques for intracranial pressure monitoring in patients with severe traumatic brain injury. J Clin Monit Comput 2011;25:231-6.

2. Brain Trauma Foundation; American Association of Neurological Surgeons; Congress of Neurological Surgeons; Joint Section on Neurotrauma and Critical Care, AANS/CNS, Bratton SL, Chestnut RM, Ghajar J, McConnell Hammond FF, Harris OA, Hartl R, Manley GT, Nemecek A, Newell DW, Rosenthal G, Schouten J, Shutter L, Timmons SD, Ullman JS, Videtta W, Wilberger JE, Wright DW. Guidelines for the management of severe traumatic brain injury. VII. Intracranial pressure monitoring technology. J Neurotrauma 2007;24 Suppl $1: S 45-54$.

3. Liu H, Wang W, Cheng F, Yuan Q, Yang J, Hu J, Ren G. External Ventricular Drains versus Intraparenchymal Intracranial Pressure Monitors in Traumatic Brain Injury: A Prospective Observational Study. World Neurosurg 2015;83:794-800.

4. O'Neill BR, Velez DA, Braxton EE, Whiting D, Oh MY. A survey of ventriculostomy and intracranial pressure monitor placement practices. Surg Neurol 2008;70:26873; discussion 273.

5. Carney N, Totten AM, O'Reilly C, Ullman JS, Hawryluk GW, Bell MJ, Bratton SL, Chesnut R, Harris OA, Kissoon N, Rubiano AM, Shutter L, Tasker RC, Vavilala MS, 
Wilberger J, Wright DW, Ghajar J. Guidelines for the Management of Severe Traumatic Brain Injury, Fourth Edition. Neurosurgery 2017;80:6-15.

6. AlAzri A, Mok K, Chankowsky J, Mullah M, Marcoux J. Placement accuracy of external ventricular drain when comparing freehand insertion to neuronavigation guidance in severe traumatic brain injury. Acta Neurochir (Wien) 2017;159:1399-411.

7. Fiorella D, Peeling L, Denice CM, Sarmiento M, Woo $\mathrm{HH}$. Integrated flat detector CT and live fluoroscopicguided external ventricular drain placement within the neuroangiography suite. J Neurointerv Surg 2014;6:457-60.

8. Krötz M, Linsenmaier U, Kanz KG, Pfeifer KJ, Mutschler W, Reiser M. Evaluation of minimally invasive percutaneous CT-controlled ventriculostomy in patients with severe head trauma. Eur Radiol 2004;14:227-33.

9. Lollis SS, Roberts DW. Robotic catheter ventriculostomy: feasibility, efficacy, and implications. J Neurosurg 2008;108:269-74.

10. Patil V, Gupta R, San Jose Estepar R, Lacson R, Cheung A, Wong JM, Popp AJ, Golby A, Ogilvy C, Vosburgh KG. Smart stylet: the development and use of a bedside external ventricular drain image-guidance system. Stereotact Funct Neurosurg 2015;93:50-8.

11. Cinalli G, Cappabianca P, de Falco R, Spennato P, Cianciulli E, Cavallo LM, Esposito F, Ruggiero C, Maggi G, de Divitiis E. Current state and future development of intracranial neuroendoscopic surgery. Expert Rev Med Devices 2005;2:351-73.

12. Theodosopoulos PV, Abosch A, McDermott MW. Intraoperative fiber-optic endoscopy for ventricular catheter insertion. Can J Neurol Sci 2001;28:56-60.

13. Ganau M, Ligarotti GK, Apostolopoulos V. Real-time intraoperative ultrasound in brain surgery: neuronavigation and use of contrast-enhanced image fusion. Quant Imaging Med Surg 2019;9:350-8.

14. Wang D, Ma D, Wong ML, Wáng YXJ. Recent advances in surgical planning \& navigation for tumor biopsy and resection. Quant Imaging Med Surg 2015;5:640-8.

15. Fichtner J, Hofmann N, Rienmüller A, Buchmann N, Gempt J, Kirschke JS, Ringel F, Meyer B, Ryang YM. Revision Rate of Misplaced Pedicle Screws of the Thoracolumbar Spine-Comparison of Three-Dimensional Fluoroscopy Navigation with Freehand Placement: A Systematic Analysis and Review of the Literature. World Neurosurgery 2018;109:e24-e32.

16. Nowacki A, Debove I, Fiechter M, Rossi F, Oertel MF, Wiest R, Schüpbach M, Pollo C. Targeting Accuracy of the
Subthalamic Nucleus in Deep Brain Stimulation Surgery: Comparison Between 3 T T2-Weighted Magnetic Resonance Imaging and Microelectrode Recording Results. Oper Neurosurg (Hagerstown) 2018;15:66-71.

17. Ann JM, Bae HG, Oh JS, Yoon SM. Device for Catheter Placement of External Ventricular Drain. J Korean Neurosurg Soc 2016;59:322-4.

18. Sarrafzadeh A, Smoll N, Schaller K. Guided (VENTRIGUIDE) versus freehand ventriculostomy: study protocol for a randomized controlled trial. Trials 2014;15:478.

19. Foreman PM, Hendrix P, Griessenauer CJ, Schmalz PG, Harrigan MR. External ventricular drain placement in the intensive care unit versus operating room: evaluation of complications and accuracy. Clin Neurol Neurosurg 2015;128:94-100.

20. Wilson MP, O'Kelly C, Jack AS, Rempel J. Utilizing preprocedural CT scans to identify patients at risk for suboptimal external ventricular drain placement with the freehand insertion technique. J Neurosurg 2018:1-7.

21. Huyette DR, Turnbow BJ, Kaufman C, Vaslow DF, Whiting BB, Oh MY. Accuracy of the freehand pass technique for ventriculostomy catheter placement: retrospective assessment using computed tomography scans. J Neurosurg 2008;108:88-91.

22. Toma AK, Camp S, Watkins LD, Grieve J, Kitchen ND. External ventricular drain insertion accuracy: is there a need for change in practice? Neurosurgery 2009;65:1197200; discussion 200-1.

23. Raabe C, Fichtner J, Beck J, Gralla J, Raabe A. Revisiting the rules for freehand ventriculostomy: a virtual reality analysis. J Neurosurg 2018;128:1250-7.

24. Ehtisham A, Taylor S, Bayless L, Klein MW, Janzen JM. Placement of external ventricular drains and intracranial pressure monitors by neurointensivists. Neurocrit Care 2009;10:241-7.

25. Srinivasan VM, O'Neill BR, Jho D, Whiting DM, Oh MY. The history of external ventricular drainage. J Neurosurg 2014;120:228-36.

26. Abdoh MG, Bekaert O, Hodel J, Diarra SM, Le Guerinel C, Nseir R, Bastuji-Garin S, Decq P. Accuracy of external ventricular drainage catheter placement. Acta Neurochir (Wien) 2012;154:153-9.

27. Hsieh CT, Chen GJ, Ma HI, Chang CF, Cheng CM, Su YH, Ju DT, Hsia CC, Chen YH, Wu HY, Liu MY. The misplacement of external ventricular drain by freehand method in emergent neurosurgery. Acta Neurol Belg 2011;111:22-8.

28. Kakarla UK, Kim LJ, Chang SW, Theodore N, Spetzler 
RF. Safety and accuracy of bedside external ventricular drain placement. Neurosurgery. 2008;63:ONS162-6.

29. Saladino A, White JB, Wijdicks EF, Lanzino G.

Malplacement of ventricular catheters by neurosurgeons: a single institution experience. Neurocrit Care 2009;10:248-52.

30. Woernle CM, Burkhardt JK, Bellut D, Krayenbuehl N, Bertalanffy H. Do iatrogenic factors bias the placement of external ventricular catheters?--a single institute experience and review of the literature. Neurol Med Chir (Tokyo) 2011;51:180-6.

31. Muirhead WR, Basu S. Trajectories for frontal external ventricular drain placement: virtual cannulation of adults with acute hydrocephalus. Br J Neurosurg 2012;26:710-6.

32. Kirkman MA, Muirhead W, Sevdalis N. The relative efficacy of 3 different freehand frontal ventriculostomy trajectories: a prospective neuronavigation-assisted simulation study. J Neurosurg 2017;126:304-11.

33. Lind CR, Correia JA, Law AJ, Kejriwal R. A survey of surgical techniques for catheterising the cerebral lateral ventricles. J Clin Neurosci 2008;15:886-90.

34. Woo H, Kang DH, Park J. Preoperative determination of ventriculostomy trajectory in ventriculoperitoneal shunt surgery using a simple modification of the standard coronal MRI. J Clin Neurosci 2013;20:1754-8.

35. Rehman T, Rehman A, Ali R, Rehman A, Bashir H, Ahmed Bhimani S, Tran H, Khan S. A radiographic analysis of ventricular trajectories. World Neurosurg 2013;80:173-8.

36. Zhang Z, Guo Q, Wang E. Hyperventilation in neurological patients: from physiology to outcome evidence. Curr Opin Anaesthesiol 2019;32:568-73.

37. Brean A, Eide PK, Stubhaug A. Comparison of intracranial pressure measured simultaneously within the brain parenchyma and cerebral ventricles. J Clin Monit Comput 2006;20:411-4.

Cite this article as: Sun Z, Wu L, Liu Z, Zhong W, Kou Z, Liu J. Optimizing accuracy of freehand cannulation of the ipsilateral ventricle for intracranial pressure monitoring in patients with brain trauma. Quant Imaging Med Surg 2020;10(11):2144-2156. doi: 10.21037/qims-20-128
38. Hayhurst C, Beems T, Jenkinson MD, Byrne P, Clark S, Kandasamy J, Goodden J, Nandoe Tewarie RD, Mallucci CL. Effect of electromagnetic-navigated shunt placement on failure rates: a prospective multicenter study. J Neurosurg 2010;113:1273-8.

39. O'Leary ST, Kole MK, Hoover DA, Hysell SE, Thomas A, Shaffrey CI. Efficacy of the Ghajar Guide revisited: a prospective study. J Neurosurg 2000;92:801-3.

40. Bogdahn U, Lau W, Hassel W, Gunreben G, Mertens HG, Brawanski A. Continuous-pressure controlled, external ventricular drainage for treatment of acute hydrocephalus-evaluation of risk factors. Neurosurgery 1992;31:898-903; discussion 903-4.

41. Dupont S, Rabinstein AA. CT evaluation of lateral ventricular dilatation after subarachnoid hemorrhage: baseline bicaudate index values [correction of balues]. Neurol Res 2013;35:103-6.

42. Bauer DF, Razdan SN, Bartolucci AA, Markert JM. Meta-analysis of hemorrhagic complications from ventriculostomy placement by neurosurgeons. Neurosurgery 2011;69:255-60.

43. Binz DD, Toussaint LG, 3rd, Friedman JA. Hemorrhagic complications of ventriculostomy placement: a metaanalysis. Neurocrit Care 2009;10:253-6.

44. Paramore CG, Turner DA. Relative risks of ventriculostomy infection and morbidity. Acta Neurochir (Wien) 1994;127:79-84.

45. Robertson FC, Abd-El-Barr MM, Mukundan S, Jr., Gormley WB. Ventriculostomy-associated hemorrhage: a risk assessment by radiographic simulation. J Neurosurg 2017;127:532-6.

46. Sabarudin A, Subramaniam C, Sun Z. Cerebral CT angiography and CT perfusion in acute stroke detection: a systematic review of diagnostic value. Quant Imaging Med Surg 2014;4:282-90. 